\title{
Chapter 6 \\ Ground-Based Measurements of Energetic Particles by Neutron Monitors
}

\author{
R. Bütikofer
}

\begin{abstract}
Since the International Geophysical Year (IGY) in 1957/58, the worldwide network of neutron monitors is the standard instrument to investigate the variations of the cosmic ray flux near Earth (11-year modulation of the galactic cosmic rays, Forbush decreases, solar cosmic ray events) in the $\mathrm{GeV}$ range. The ensemble of neutron monitors together with the geomagnetic field acts as a giant spectrometer and enables to deduce information about the primary cosmic ray spectrum near Earth in the energy range $\sim 500 \mathrm{MeV}$ to $\sim 15 \mathrm{GeV}$. For the interpretation of the ground-based neutron monitor measurements, the transport of the cosmic rays in the Earth's magnetic field as well as the transport in the Earth's atmosphere and the detection efficiency of the secondary nucleons by the neutron monitors must be known. The Neutron Monitor Data Base (NMDB) developed in 2008/09 enables a rapid accessibility to the data of the worldwide neutron monitor network. A considerable number of neutron monitor stations send their data to NMDB in real-time which enables the operation of space weather applications based on neutron monitor data.
\end{abstract}

\subsection{Introduction}

The ground-based neutron monitors are relatively simple instruments in respect to technology and electronics. They are ideally suited to measure the intensity of the nucleonic component of the secondary cosmic radiation in the Earth's atmosphere and respond to primary cosmic ray particles in the GeV-range. Even after 60 years of operation the neutron monitors remain the state-of-the-art instrument for measuring the intensity variations of the primary cosmic rays in the energy range from $\sim 500 \mathrm{MeV}$ to $\sim 30 \mathrm{GeV}$. This energy region complements the range above the energies covered by space-based cosmic ray detectors. The worldwide network of

\footnotetext{
R. Bütikofer $(\bowtie)$

University of Bern, Physikalisches Institut, Sidlerstrasse 5, CH-3012 Bern, Switzerland

High Altitude Research Stations Jungfraujoch and Gornergrat, Sidlerstrasse 5, CH-3012 Bern, Switzerland

e-mail: rolf.buetikofer@space.unibe.ch
}

(C) The Author(s) 2018

O.E. Malandraki, N.B. Crosby (eds.), Solar Particle Radiation Storms Forecasting and Analysis, The HESPERIA HORIZON 2020 Project and Beyond, Astrophysics and Space Science Library 444, DOI 10.1007/978-3-319-60051-2_6 
neutron monitors is an excellent tool to investigate variations of the primary cosmic ray flux near Earth such as 11-year modulation and the sudden transient effects as Forbush decreases ${ }^{1}$ and solar cosmic ray events. Since recently, neutron monitor measurements are also an important input for space weather applications.

In the International Geophysical Year 1957/58 the worldwide network of standardised neutron monitors was developed to investigate the variations of the cosmic ray intensity near Earth. There are two types of standardised neutron monitors in operation. The IGY (for International Geophysical Year) type was designed by Simpson (1955) in the early 1950s. About 10 years later Carmichael (1968) designed the larger NM64 monitor with an increased counting rate. Figure 6.1 shows the 18-IGY neutron monitor at Jungfraujoch, Switzerland (left) and the 6-NM64 monitor of Athens, Greece (right). The digits 18 respectively 6 give the number of the counter tubes deployed in the respective neutron monitor.

The ensemble of neutron monitors together with the geomagnetic field acts as a giant spectrometer and enables the determination of the spectral variations of the galactic cosmic rays near Earth and the spectral characteristics of the sporadic solar cosmic rays. In addition, the simultaneous detection of relativistic particles with the entire global network of neutron monitors provides information about the anisotropy of the cosmic ray flux near Earth as the viewing directions of each neutron monitor station at the border of the geomagnetosphere depends on the neutron monitor's location, on the cosmic ray particle's rigidity, and on the direction of incidence above the neutron monitor station.

To deduce the variation of the primary cosmic rays near Earth but outside the geomagnetosphere from neutron monitor measurements, the relationship between the neutron monitor count rate and the primary cosmic ray flux must be known.
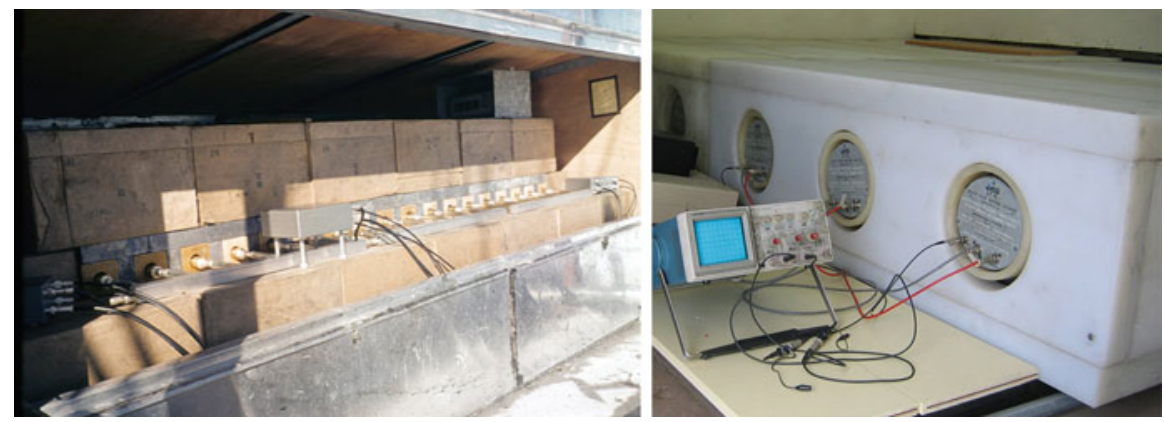

Fig. 6.1 18-IGY neutron monitor Jungfraujoch, Switzerland, (left) and 6-NM64 neutron monitor Athens, Greece (right). The digits 18 respectively 6 give the number of the counter tubes of the corresponding neutron monitor station

\footnotetext{
${ }^{1}$ Decrease within hours in the galactic cosmic ray intensity near Earth caused by the passage of a coronal mass ejection (CME) and slow recovery within days, named after the American cosmic ray physicist Scott E. Forbush.
} 
When primary cosmic ray particles approach the Earth, they enter first the geomagnetosphere, where the cosmic ray particles are deviated by the Earth's magnetic field (Lorentz force) and then penetrate into the Earth's atmosphere, where the cosmic ray particles make electromagnetic interactions with the atoms and molecules as well as hadronic processes with the nuclei of the atmospheric constituents. A cascade of various secondary particles is produced.

In Sect. 6.2 a short overview of the history of the neutron monitors is given. The transport of cosmic rays in the Earth's magnetic field is described in more detail in Chap. 5. The transport of cosmic ray particles through the Earth's atmosphere is addressed in Sect. 6.3. The neutron monitor, i.e. its structure, layout, the functions of the different parts of the detector, the response of the neutron monitor to primary cosmic rays, and environmental effects on the measurements are described in Sect. 6.4. As a single neutron monitor does not give information about the energy spectrum and the direction of the flux of the primary cosmic rays, a network of neutron monitors at different latitudes and longitudes is needed to retrieve this information. Today this network contains about 50 operating stations. Section 6.5 gives an overview about the worldwide network of neutron monitor stations. The neutron monitor database NMDB initiated in 2008/09 as an European FP7 project is presented in Sect. 6.6.

\subsection{History}

After the discovery of the cosmic rays in 1912 by Victor Hess, mainly ionisation chambers on ground were used to investigate the variations of the cosmic ray intensities. The basic ideas for the development of neutron monitors as a continuous recorder of the cosmic ray intensity originated from the measurements by Simpson (1948). He found that the latitude dependences of the intensities of the energetic nucleonic component and of the evaporation neutrons from the secondary cosmic rays in the atmosphere are several times larger than those of the ionising component (ionisation chambers) and the hard component (muon counters). In addition, the measurement of the nucleonic component allows to study the time variations of the primary cosmic rays at lower energies than this is possible with ionisation chambers or muon counters. These facts stimulated the development of new detectors that measure the secondary neutrons in the atmosphere.

The neutron monitor designed by Simpson (1955) was adopted as the standard detector during the International Geophysical Year (IGY) 1957/58 and was called IGY neutron monitor. It became evident soon that better statistical accuracy was required, in particular for the study of short-term events as e.g. solar cosmic ray events, so-called GLEs (Ground Level Enhancements or Ground Level Events). In 1959 large sized proportional counter tubes were constructed and produced at the Chalk River Nuclear Laboratories, Ontario, Canada. This led to the design and the construction of the supermonitor or NM64 monitor for the International Quiet Sun Year (IQSY) (Carmichael 1968). The counting rate of the NM64 monitor per unit 
area of lead producer is about three times that of the IGY neutron monitor. Today mainly NM64 monitors are in operation.

The different neutron monitor stations are mostly operated by research institutions that are located near the stations. For the data exchange in the days of the advent of neutron monitors the operators of the stations sent their hourly data in the form of tables on paper by mail to the World Data Centers (WDCs) (Pyle 2000), later on magnetic tapes and afterward on floppy disks. With the internet the data exchange became much easier.

\subsection{Transport of Cosmic Ray Particles in the Earth's Atmosphere}

Primary cosmic ray particles that penetrate the atmosphere undergo multiple interactions resulting in showers of secondary particles. If the secondary nucleons (neutrons or protons) reach the ground, they can be detected by neutron monitors.

To deduce the cosmic ray characteristics at the top of the atmosphere from neutron monitor measurements, the transport in the atmosphere, i.e. the interactions of energetic particles with matter, and the detection efficiency of the neutron monitor must be known. The physics of the interactions in the atmosphere when cosmic ray particles enter the atmosphere are today usually simulated with Monte Carlo methods.

The Earth's atmosphere, i.e. the medium in which the interactions take place, is described by a model (Sect. 6.3.1). The essential nuclear interactions of the cosmic ray particles when entering into the atmosphere, which are relevant for ground-based neutron monitor measurements, are addressed in Sect. 6.3.2.

\subsubsection{Model of the Earth's Atmosphere}

There exist several models that describe the properties (pressure, temperature, density, chemical composition) of the Earth's atmosphere primarily as a function of altitude (US Standard Atmosphere, International Standard Atmosphere, NRLMSISE-00).

Within the Geant4 (Agostinelli et al. 2003) software PLANETOCOSMICS (Desorgher et al. 2006), which is often used to simulate the transport of cosmic ray particles in the atmosphere, it is possible to select between the MSISE-90 model and its upgraded version NRLMSISE-00 (Labitzke et al. 1985; Hedin 1991; Picone et al. 2002). MSIS stands for Mass Spectrometer and Incoherent Scatter Radar, $\mathrm{E}$ indicates that the model extends from the ground through the exosphere and the number at the end of the short name is the year of release. NRL stands for the US Naval Research Laboratory. Both models provide temperature, density and 
concentration profiles vs. altitude from the ground to the exobase $(\sim 450-500 \mathrm{~km})$ as function of geographic latitude, longitude, date and time in UT, $F_{10.7}$ index $(10.7 \mathrm{~cm}$ solar radio flux used as solar UV proxy ), $F_{10.7 A}$ index ( 3 month average of $F_{10.7}$ ) and the geomagnetic index $A p$. The dependence of the model on $F_{10.7}, F_{10.7 A}$, and $A p$ is neglectable below $80 \mathrm{~km}$. The Earth's atmosphere is divided into superposed homogeneous layers above a solid Earth. The density and composition of the layers are computed according to the altitude or atmospheric depth from the atmospheric model and are constant throughout each layer. The thickness of the layers may be selected by the user. The models may take different geometries: flat or concentric spherical geometry. In the case of the spherical geometry the Earth is modeled by a sphere of $6371.2 \mathrm{~km}$ radius and overlying curved layers.

\subsubsection{Particle Cascade in the Atmosphere}

When primary cosmic ray particles enter the Earth's atmosphere, they make electromagnetic interactions with the atoms and molecules of the atmospheric gases and hadronic interactions with the nuclei of the atmospheric matter. Thereby the cosmic ray particles rapidly loose energy and produce various secondary particles. The mean free path for nuclear interactions of a cosmic ray particle (proton) with nitrogen or oxygen nucleus is $\sim 75 \mathrm{~g} / \mathrm{cm}^{2}$.

For the interpretation of neutron monitor measurements the nucleonic or hadron component in the atmosphere is relevant. The products of the nucleonic interactions are secondary nucleons and pions $\left(\pi^{+}, \pi^{-}\right.$, and $\left.\pi^{0}\right)$. The secondary protons lose their energy mainly by ionisation. When the secondary nucleons have sufficient energy, they continue to multiply in successive generations of nuclear collisions until the energy of the particles drops below the energy that is required for multiple pion production, i.e. about $1 \mathrm{GeV}$. Secondary protons and ions with energies $\leq 100 \mathrm{MeV}$ no longer undergo hadronic interactions, they are rapidly decelerated to rest by ionisation. On the other hand the neutrons still make nuclear interactions at these energies as well as elastic collisions with nuclei in the atmosphere. Below $10 \mathrm{MeV}$ the neutrons lose their energy continuously by elastic collisions with atmospheric nuclei before they are captured by nucleons at thermal energies.

The neutral pions $\pi^{0}$ have a very short mean lifetime $\tau=1.78 \times 10^{-16} \mathrm{~s}$. The $\pi^{0}$ decays immediately into two $\gamma$-rays which initiate an electromagnetic cascade. The charged pions $\pi^{+}$and $\pi^{-}$decay into muons $\pi^{+} \rightarrow \mu^{+}+v_{\mu}$ and $\pi^{-} \rightarrow \mu^{-}+\bar{v}_{\mu}$ with a mean lifetime of $2.55 \times 10^{-8} \mathrm{~s}$. The muons are slowed down mainly by ionisation. The low energy muons have time to decay $\left(\tau=2.2 \times 10^{-6} \mathrm{~s}\right)$ before they reach the ground. The reactions are $\mu^{+} \rightarrow e^{+}+v_{e}+\bar{v}_{\mu}$ and $\mu^{-} \rightarrow e^{-}+\bar{v}_{e}+v_{\mu}$. However, many of the muons are produced with very high energies in the uppermost layers of the atmosphere and as these muons loose only little energy they have a large path length and survive (time dilatation according to the theory of relativity) and reach the surface of the Earth. The muons deep in the atmosphere and at sea level 
are the most dominant component of the secondary cosmic rays and are therefore the dominant source of ionisation in this altitude range.

The primary particles at the top of the atmosphere must have an energy of roughly $500 \mathrm{MeV}$ per nucleon to produce a cascade of secondary nucleons that can reach the ground at sea level. In high latitude regions, where the shielding effect of the Earth's magnetic field for incident cosmic rays is low, the lower threshold of the neutron monitor response is controlled therefore by the atmospheric mass which is $\sim 1030 \mathrm{~g} \mathrm{~cm}^{-2}$ at sea level. For high latitude neutron monitors at sea level this atmospheric cutoff for primary cosmic rays is $\sim 450 \mathrm{MeV} /$ nucleon. In regions like Central Antarctica, at an elevation of $\sim 3 \mathrm{~km}$ above sea level (asl), the reduced atmospheric mass lowers the threshold to $\sim 300 \mathrm{MeV} /$ nucleon (Mishev et al. 2014).

\subsection{Neutron Monitor Detector}

Neutron monitors cover the energy range of primary cosmic ray particles from $\sim 0.5$ to $\gtrsim 100 \mathrm{GeV}$ per nucleon. This energy range includes the solar modulation of the galactic cosmic rays, Forbush decreases, sporadic GLEs or relativistic SEP (Solar Energetic Particle) events, and geomagnetic effects. The longterm stability of neutron monitors is generally excellent so that the cosmic ray effects of the 11-year solar activity cycle can be investigated over several solar cycles. The longest time series of single neutron monitor stations are available over a period of $\sim 60$ years, i.e. during a time range over more than five solar activity cycles. The comparison of the measurements of different neutron monitor stations have however shown that some neutron monitors may show a degrading of the detector efficiency (Bieber et al. 2007).

As the name suggests, neutron monitors record predominantly the secondary neutrons from the atmospheric cascades. The contribution to the total neutron monitor counting rate of an NM64 are neutrons $\sim 85 \%$, protons $\sim 7 \%$, $\mu \mathrm{s} \sim 6 \%$, $\pi \mathrm{s} \sim 1 \%$ (Hatton 1971).

The functionality, construction and other properties of a neutron monitor are described in Sect.6.4.1. The response of the neutron monitor to primary cosmic ray particles at the top of the Earth's atmosphere is explained in Sect. 6.4.2. The influence of the atmospheric effects on the neutron monitor measurements are addressed in Sect. 6.4.3.

\subsubsection{Components of a Neutron Monitor}

Both neutron monitor types IGY (Simpson 1955) and NM64 (Carmichael 1964) employ the same measurement strategy, i.e. the difference in the way high and low energy neutrons interact with different nuclei. As a particle with no electric charge, the neutron makes only interactions with nuclei and can therefore penetrate 
large layers of material without interactions because of the small range of the strong nuclear force. Energetic neutrons can make three different kinds of interactions with nuclei: elastic and inelastic collisions as well as nuclear reactions. After a hadronic interaction of an energetic neutron with a nucleus, the excited target nucleus emits so-called evaporation neutrons. The production of these evaporation neutrons is proportional to $A^{2 / 3}$ according to the nuclear physics theory, where $A$ is the atomic weight of the target nucleus. In a material containing nuclei with low atomic mass, the neutrons are effectively slowed down (moderated) in elastic collisions.

These facts led Simpson to the neutron monitor detector concept: production of fast neutrons in a target with high atomic weight, braking of the fast neutrons in a hydrogenous material, and finally detection of the thermic neutrons indirectly by ionising particles that are produced in a neutron induced nuclear reaction.

Figure 6.2 shows a schematic diagram of the NM64 neutron monitor. A standard NM64 neutron monitor with six counter tubes (6-NM64) has the following dimensions: width: $\sim 315 \mathrm{~cm}$, depth: $\sim 220 \mathrm{~cm}$, and height: $\sim 50 \mathrm{~cm}$. The lead producer with $\sim 9650 \mathrm{~kg}$ is by weight the major component of an 6-NM64 monitor.

The different components of a neutron monitor detector are described in the following. The given specifications are valid for the NM64 neutron monitor (Carmichael 1964):

Reflector The whole assembly of the detector is enclosed by polyethylene (protonrich material) of an average thickness of $7.5 \mathrm{~cm}$. The task of the reflector is to reflect and to moderate the evaporation neutrons that are produced in the lead producer. The polyethylene (moderator material) contains a significant fraction of hydrogen, i.e. the energy loss per elastic collision of a neutron with the moderator material is maximal as the mass of the projectile and of the hydrogen nuclei in the target material are almost equal (conservation of momentum and energy). The neutron elastic interaction pathlength with hydrogen in polyethylene is roughly $1 \mathrm{~cm}$ for

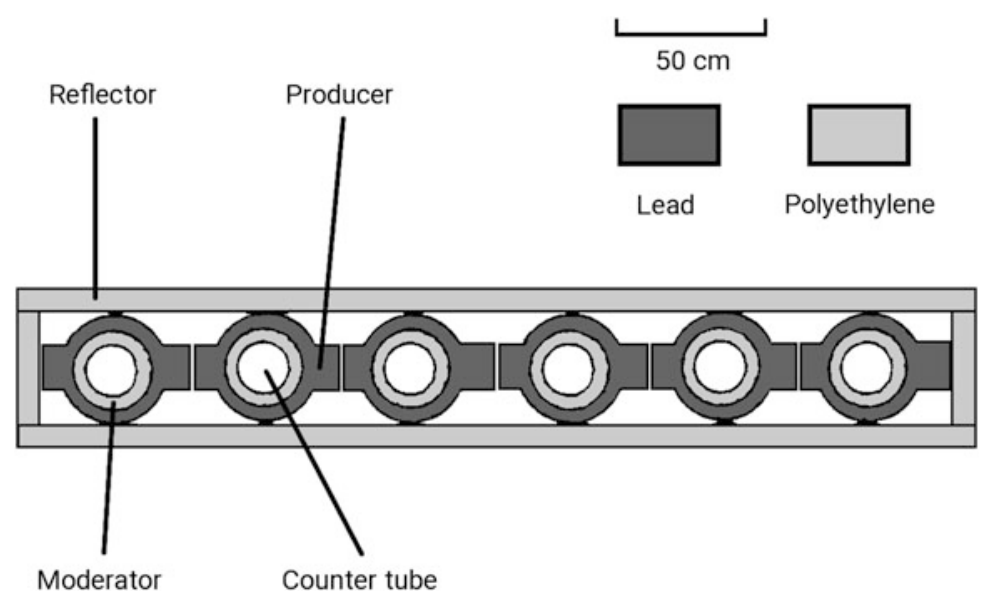

Fig. 6.2 Schematic view of a 6-NM64 neutron monitor 
neutrons with energies $\leq 1 \mathrm{MeV}$ and in each collision the incident neutron reduces its kinetic energy on average by a factor of 2 , i.e. the evaporation neutrons are very effectively slowed down in the reflector.

In addition, this neutron monitor component has the function to reflect and to absorb the low energy neutrons that are produced by high energy nucleons in interactions with the ambient material of the neutron monitor, e.g. detector housing.

In contrast, this reflector is largely transparent to the energetic neutrons that are produced in the cosmic ray induced cascade in the atmosphere, i.e. these energetic neutrons can easily reach and enter the lead producer.

Producer The core of the neutron monitor consists of a lead producer, a target with high atomic mass $(A)$, to produce secondary neutrons. The average depth of $13.8 \mathrm{~cm}\left(156 \mathrm{~g} / \mathrm{cm}^{2}\right)$ corresponds to about $75 \%$ of the inelastic mean-free path of nucleons in lead. Thus $\sim 50 \%$ of the nucleons, that cross the reflector and enter the lead, make at least one interaction in the producer. The production rate of neutrons per inelastic nucleon-nucleus interaction is roughly proportional to $A^{0.7}$ in the energy range $100-700 \mathrm{MeV}$ of the interacting nucleon and slowly decreases with increasing energy (Shen 1968). The average number of produced neutrons (multiplicity) depends weakly on the incident nucleon energy, i.e. a neutron monitor can be used as an energy spectrometer by measuring the multiplicity only limited. On average about 15 evaporation neutrons with mean energy $\sim 2.5 \mathrm{MeV}$ are produced per nuclear reaction (Hatton 1971). These neutrons amplify the cosmic ray signal and can not easily escape the reflector. The lead producer is interspersed with a moderator and the $\mathrm{BF}_{3}$ proportional counter tubes.

Moderator Each counter tube is surrounded by a polyethylene tube with a thickness of $2 \mathrm{~cm}$ acting as a moderator for the evaporation neutrons that are generated in the lead producer.

Proportional Counter The proportional counter tubes are filled with $\mathrm{BF}_{3}$ (boron trifluoride) as counter gas. The $\mathrm{BF}_{3}$ has been $90 \%$ enriched with the ${ }^{10} \mathrm{~B}$ isotope. When the very slow neutrons (thermal neutrons, $E=\frac{3}{2} k T=\sim 0.04 \mathrm{eV}$ ) encounter $\mathrm{a}^{10} \mathrm{~B}_{5}$ nucleus in the proportional counter, the following favoured reaction may take place:

$$
{ }^{10} \mathrm{~B}_{5}+\mathrm{n} \rightarrow{ }^{7} \mathrm{Li}_{3}+\alpha
$$

The cross-section for this reaction is inversely proportional to the neutron speed and has a value of about $3.0 \cdot 10^{-25} \mathrm{~m}^{2}$ or 3000 barns at neutron energy $0.04 \mathrm{eV}$ and only roughly 0.2 barns at $1 \mathrm{MeV}$ (Clem and Dorman 2000). The produced $\alpha$-particle and the Li-nucleus are accelerated by the applied high voltage within the counter tube, ionize the counter gas and the produced electrons cause an electric signal. The electric signal is amplified, discriminated and counted by a counter electronic. The detection probability of the evaporation neutrons is $\sim 5.7 \%$ (Hatton 1971).

Later proportional counter tubes were filled with ${ }^{3} \mathrm{He}$ as an alternative to the standard $\mathrm{BF}_{3}$ counters, as $\mathrm{BF}_{3}$ is highly toxic. The He counters require a higher 
pressure to have an efficiency close to the $\mathrm{BF}_{3}$ counters and they have a much higher temperature sensitivity and therefore require better environmental temperature stability. Currently, new neutron monitor counter tubes use again $\mathrm{BF}_{3}$ because of the very high price for ${ }^{3} \mathrm{He}$.

As an incident neutron or proton into the neutron monitor may produce more than one evaporation neutron in the lead producer, it can be expected that a group of count signals is observed (multiplicity). However, the efficiency for detecting these evaporation neutrons is low. Therefore, the average detected multiplicity is typically not much larger than one. Due to the multiplicity effect in the neutron monitor the count impulses are not equally distributed. Therefore the variance of the counting rate $\sigma(N)$ is not Poisson distributed. One has $\sigma(N)>\sqrt{N}$. The variance of the counting rate $\sigma(N)$ is given by:

$$
\sigma(N)=k \cdot \sqrt{N}
$$

where $k$ is around 1.5 for an NM64 monitor (Hatton 1971).

The average count rate of an 6-NM64 monitor at high latitude and at sea level is $\sim 4200$ counts per minute. Due to the multiplicity effect in the neutron monitor the relative random error for 1-min values is therefore $\sim 2.5 \%$. The count rate of an equatorial sea-level 6-NM64 monitor is $\sim 0.7$ times the count rate of an identical neutron monitor at high latitudes. Neutron monitors at high altitudes have higher counting rates because of the smaller atmospheric attenuation. The count rate of a neutron monitor at high latitudes and at an altitude of $\sim 3000 \mathrm{~m}$ asl is about a factor of ten higher than at sea level.

\subsubsection{Neutron Monitor Yield Function}

The transport of cosmic ray particles through the Earth's atmosphere and the detection of the nucleonic component of the secondary cosmic rays by the neutron monitors are combined in the so-called neutron monitor yield function. The neutron monitor yield function can therefore directly be used to determine the cosmic ray flux at the top of the Earth's atmosphere from the measurements of the worldwide neutron monitors. Essentially, two methods are used to determine the neutron monitor response function:

- parameterisation of latitude survey observations (neutron monitor measurements e.g. on a ship cruise along a large range of geomagnetic latitudes)

- Monte Carlo simulations of the cosmic ray transport through the Earth's atmosphere and of the detection efficiency for the different secondary particles in the neutron monitor

The most commonly used response function based on latitude surveys is the Dorman function (Dorman and Yanke 1981). The Dorman function represents most latitude surveys fairly well, however the trend of the response function at low rigidities 
can not be retrieved from latitude surveys. Belov and Struminsky (1987) made modifications to the Dorman function for rigidities $<2.78 \mathrm{GV}$.

Different authors have determined the neutron monitor yield function with Monte Carlo simulations. In 1982 Debrunner et al. calculated the specific yield function for sea level neutron monitors. Clem and Dorman (2000) applied the FLUKA Monte-Carlo package (Fassò et al. 1993) for the simulations. In recent years different groups (Flückiger et al. 2008; Matthiä 2009; Mishev et al. 2013) used the Geant4 software package (Agostinelli et al. 2003) to compute the neutron monitor yield function. For the transport in the atmosphere the Geant 4 software suite PLANETOCOSMICS (Desorgher et al. 2006) has been mostly used. Some of the determined yield functions are valid only for neutron monitors at sea level. This requires pressure corrections of the neutron monitor count rates to sea level. Mainly for high altitude neutron monitor stations these corrections may be inaccurate as the parameter for the barometric corrections depends on the cosmic ray spectrum, see Sect. 6.4.3. The yield function by Flückiger et al. (2008) is valid for different altitudes, i.e. the atmospheric depth of the neutron monitor station is a parameter of this yield function.

The relation between the counting rate $N_{x}$ of the neutron monitor station $x$ and the differential fluxes of the different components $k$ of the primary cosmic rays at the border of the geomagnetic field, $\psi_{k}$, can be described by the following formula:

$$
\begin{aligned}
N_{x}(h, \Lambda, \chi, t)= & \sum_{k} \int_{0}^{2 \pi} \int_{0}^{\pi / 2} \int_{0}^{\infty} \alpha(R, \theta, \phi, \Lambda, \chi, t) \cdot \psi_{k}(R, \theta, \phi, \Lambda, \chi, t) . \\
& \sin \theta \cdot S_{x}^{k}(h, R, \theta) \cdot A_{x} \cdot \cos \theta \cdot d R d \theta d \phi
\end{aligned}
$$

where

$\alpha \quad$ filter function which is 1 for allowed cosmic ray particle trajectories and 0 for forbidden trajectories (Chap. 5).

$R \quad$ rigidity of the primary cosmic ray particle. $R=\frac{p \cdot c}{Z \cdot e}$ where $p$ is the momentum of the particle, $Z e$ is its charge and $c$ is the speed of light.

$\theta, \phi \quad$ zenith and azimuth angle of the primary cosmic rays at the top of the atmosphere.

$\Lambda, \chi \quad$ geographic latitude and longitude of the neutron monitor station location.

$t \quad$ date and time in UT.

$S_{x}^{k} \quad$ yield function. It gives the number of count events produced by a primary particle of type $k$ in the neutron monitor station $x$. The yield function depends on the rigidity, on the zenith angle, $\theta$, of the incident particle, and on the atmospheric depth, $h$, of the neutron monitor station.

$\mathrm{h} \quad$ atmospheric depth of the neutron monitor station.

$A_{x} \quad$ area of the neutron monitor station, $x$. 


\subsubsection{Atmospheric Effects}

Because of the interactions of the primary and secondary cosmic ray particles with the matter of the Earth's atmosphere, the neutron monitor count rate depends also on meteorological conditions (Carmichael et al. 1968). With constant cosmic ray intensity at the top of the Earth's atmosphere the counting rate of a neutron monitor depends mainly on the atmospheric mass above the detector and in much lower degree on the temperature profile and on the water content in the atmosphere. Because of the relative small effects and because of the large complexity to determine the temperature profile and the water content in the atmosphere, only the change in the atmospheric mass is considered for neutron monitor measurements. In practice, the barometric pressure is used as a proxy for the air mass to correct the neutron monitor data to a constant atmospheric depth.

The dependence of the neutron monitor count rate upon atmospheric pressure is usually described by an exponential function:

$$
N(p(t))=N(\bar{p}) \cdot \exp \left(\frac{\bar{p}-p(t)}{\lambda}\right)
$$

where

$N(p(t)) \quad$ measured count rate at atmospheric pressure $p$ and at time $t$.

$N(\bar{p}) \quad$ neutron monitor count rate at some standard pressure $\bar{p}$.

$\lambda \quad$ attenuation length of the nucleonic component of the cosmic radiation in the Earth's atmosphere.

The attenuation length $\lambda$ depends on the altitude, the geomagnetic latitude and on the primary cosmic ray spectrum. The attenuation length is larger for harder primary rigidity spectra and vice versa.

During a solar cosmic ray event measured by neutron monitors, the cosmic ray near Earth includes a galactic and a solar component. The solar particles show a softer rigidity spectrum compared to the spectrum of the galactic cosmic rays. The attenuation length for galactic cosmic rays $\lambda_{g}$ is about $140 \mathrm{~g} / \mathrm{cm}^{2}$, whereas the value for the solar cosmic rays $\lambda_{s}$ is typically around $100 \mathrm{~g} / \mathrm{cm}^{2}$. The two-attenuation length method by McCracken (1962) considers this fact.

The pressure corrected neutron monitor counting rate to the selected standard pressure $\bar{p}$ during a solar cosmic ray event can be written as follows by assuming that the galactic cosmic ray intensity during the solar cosmic ray event does not change:

$$
\begin{aligned}
N(t, \bar{p})= & N_{0}\left(p_{0}\right) \cdot \exp \left(\frac{p_{0}-\bar{p}}{\lambda_{g}}\right)+ \\
& {\left[N(t, p(t))-N_{0}\left(p_{0}\right) \cdot \exp \left(\frac{p_{0}-p(t)}{\lambda_{g}}\right)\right] \cdot \exp \left(\frac{p(t)-\bar{p}}{\lambda_{s}}\right) }
\end{aligned}
$$


where

$N(t, \bar{p}) \quad$ pressure corrected count rate to the standard pressure $\bar{p}$ of the neutron monitor station during a solar cosmic ray event.

$N_{0}\left(p_{0}\right) \quad$ average measured count rate with an average atmospheric pressure $p_{0}$ during the reference time interval (typically the full hour before the onset of the solar cosmic ray event).

$\lambda_{g} \quad$ attenuation length for the nucleonic component of the galactic cosmic rays in the Earth's atmosphere.

$N(t, p(t)) \quad$ measured count rate at time $t$ and atmospheric pressure $p(t)$.

$\lambda_{s} \quad$ attenuation length for the nucleonic component of the solar cosmic rays in the Earth's atmosphere.

The first summand of equation (6.5) is the contribution to the count rate by the galactic cosmic ray corrected to the standard pressure $\bar{p}$. The second summand is the pressure corrected count rate caused by the solar cosmic rays. The expression between square brackets is the measured part to the counting rate caused by the solar cosmic ray at time $t$. For the determination of this portion the measured counting rate during the reference time interval $N_{0}\left(p_{0}\right)$ has to be corrected to the current atmospheric pressure $p(t)$ and has to be subtracted from the measured count rate $N(t, p(t))$ during the GLE.

The barometric pressure coefficient for galactic cosmic rays $\alpha=\lambda^{-1}$ for a neutron monitor has a value in the order of $1 \% / \mathrm{mmHg}$ (or $0.0072 \mathrm{mbar}^{-1}$ ), i.e. the change in the air mass above a neutron monitor station has a large effect upon the count rate. Therefore, the barometric pressure at a neutron monitor station must be determined very accurately, as an error in the pressure measurement of $1 \mathrm{mmHg}$ causes a change in the count rate of $\sim 1 \%$. As the spectrum of the galactic cosmic ray changes during the 11-year solar activity cycle, the barometric coefficient $\alpha$ shows a variation as well and should therefore be determined periodically.

Neutron monitor stations at exposed locations (e.g. high altitudes) may considerably be affected by environmental effects. These neutron monitor stations are heavily exposed to high wind speeds and gusty winds which may strongly affect the atmospheric pressure measurements. Consequently, the correction of the neutron monitor count rates for the effects of changes in the air mass above the detector using raw barometer data may lead to erroneous results (Bütikofer and Flückiger 1999). In addition, there are other environmental effects on the neutron monitor counting rate, e.g. the accumulation of snow on the roof and around the detector housing. This effect must usually not be taken into account during a solar cosmic ray event. However, when investigating long time data series, the use of neutron monitor stations, where possible changes of snow accumulations may occur on the roof and around of the detector housing, must be considered with care. These effects must be considered especially for the NM64 type as the thickness of the reflector is only $7.5 \mathrm{~cm}$ compared to $28 \mathrm{~cm}$ in the IGY neutron monitor. In the NM64 monitor the evaporation neutrons, that are produced in the surrounding material of the neutron 
monitor, contribute to the counting rate with $\sim 5 \%$ (Hatton 1971) and changes of this matter in the immediate environment therefore affect the counting rate.

\subsection{Worldwide Network of Neutron Monitor Stations as a Giant Spectrometer}

The 'Simpson' neutron monitor (Simpson 1955) was the standard cosmic ray detector for the International Geophysical Year (IGY) 1957/58, and it was called the 'IGY' neutron monitor. During the years 1957-1959 a worldwide network of 51 monitors was established. After the International Geophysical Year 1957/58 some of the IGY neutron monitors stopped, however most stations continued operating. With the launch of the NM64 or 'supermonitor' in the 1960s by Carmichael (1968) many of the IGY neutron monitors were replaced by the new detector type. During this transition time most principal investigators operated both neutron monitor types in parallel for some months to determine a normalization factor for long term studies. Today the majority of the worldwide network comprises NM64 monitors, however there are still a few IGY neutron monitors in operation. Figure 6.3 shows a world map with the locations of the neutron monitor stations that have been in operation in 2017 or only recently been closed. In 2017 about 50 neutron monitor stations have been in operation.

The Earth's magnetic field establishes the worldwide network of neutron monitors to a huge spectrometer. The rigidity range of this spectrometer is determined by the atmospheric cutoff at the lower rigidity border and by the highest magnetic cutoff rigidity at the other end. Although the magnetic cutoff rigidity near the geomagnetic

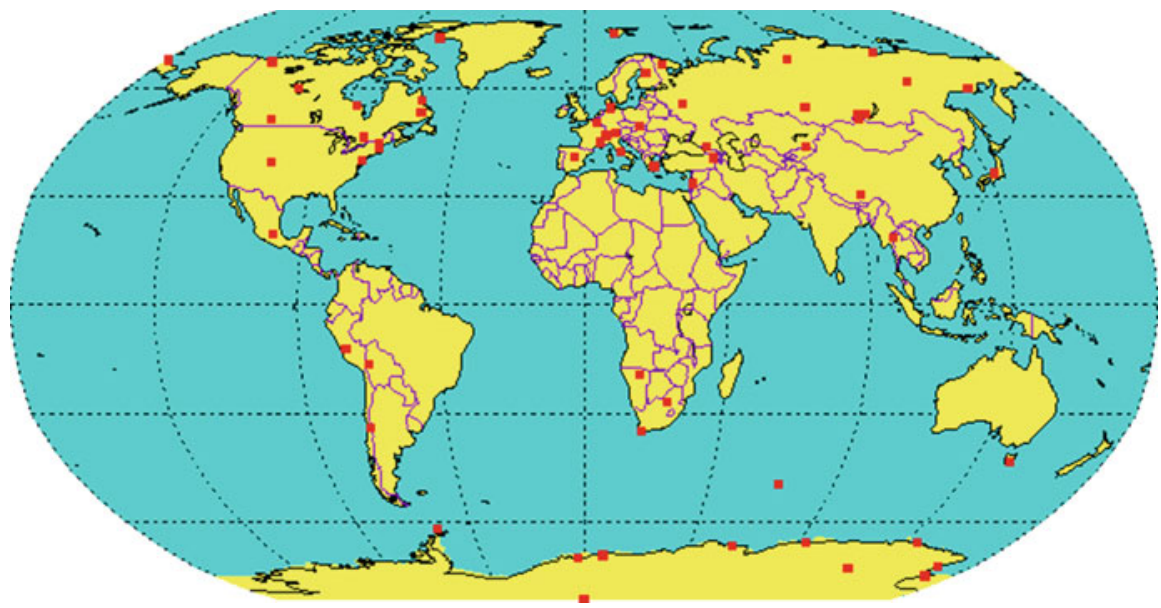

Fig. 6.3 World map with the locations of neutron monitors which have been in operation in 2017 or only recently been closed 
poles is $0 \mathrm{GV}$ as the magnetic field lines enter vertically into the Earth, the primary cosmic ray particles penetrating the top of the atmosphere must have a minimal energy that the secondary nucleons can reach the ground. This atmospheric cutoff energy for a sea level detector is $\sim 450 \mathrm{MeV}$, i.e. a rigidity of $\sim 1 \mathrm{GV}$ for protons. The maximum vertical magnetic cutoff rigidity is $\sim 15 \mathrm{GV}$. The measurements of the worldwide network of neutron monitors enable therefore to determine spectral variations of the galactic cosmic rays near Earth and the spectral characteristics of GLEs in the energy range from $\sim 500 \mathrm{MeV}$ to $\sim 15 \mathrm{GeV}$.

When working with neutron monitor data it is important to realise that the neutron monitor stations of the worldwide network are operated by different institutes, i.e. the measurements (e.g. obvious outliers in the count rate, data gaps etc.) are handled differently. The neutron monitor stations are located at very different locations (sea level, high altitude, polar regions), therefore e.g. the stability of the temperature inside the detector housing, of the humidity as well as the behaviour of the electronic devices may differ and cause different qualities of the measurements. In earlier days there were also problems with the accuracy of the used clocks.

\subsection{Neutron Monitor Database: NMDB}

In the early days of neutron monitors, the cosmic ray scientists exchanged their data with data tables and books (mostly on a monthly/half-year basis and only with a resolution of one hour). Later the operators of neutron monitor stations wrote the measured data on storage media like magnetic tapes or floppy disks and sent these media to the World Data Centers (WDC) in the USA, USSR, and Japan (Pyle 2000). For neutron monitor data analysis the scientists ordered the data either directly from the PIs or from the WDCs and received the data on magnetic tape and later on compact discs. The advent of the internet made the data exchange much easier. The different groups published their measurements on their own webpage. More and more the cosmic ray scientists have the demand to have the measurements of the worldwide network available in real-time. There were also some initiatives to develop a data base for neutron monitor data, however only the project "NMDB - Real-Time database for high resolution Neutron Monitor measurements" (http: Iwww.nmdb.eu) funded by the Commission of the European Communities as an FP7 project in the years 2008/09 was successful. A number of 12 institutions were involved in the project. First only the data of the institutions involved in the project were brought into NMDB. However, since then the number of neutron monitor stations that send their data to the NMDB has increased and in 2017 a total of about 40 neutron monitor stations transmit their data regularly to NMDB, about 30 neutron monitor stations in real-time or near real-time. The neutron monitor measurements are stored in NMDB as 1-min and hourly data. 


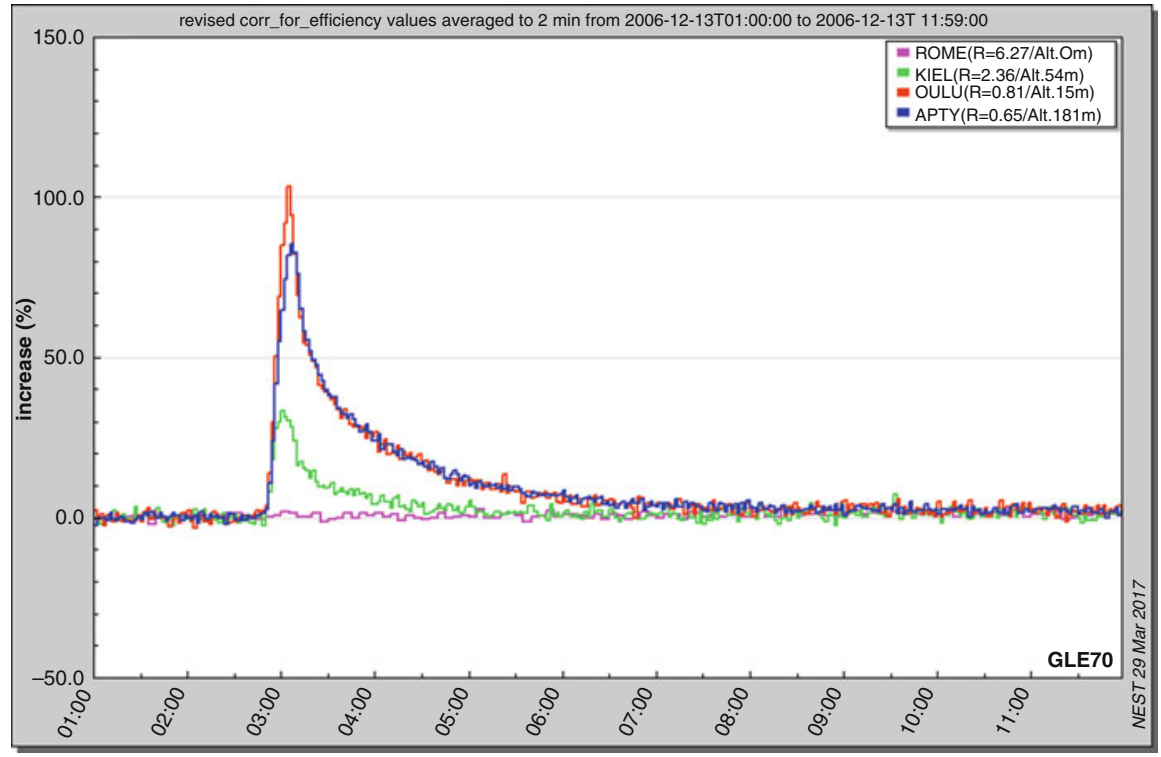

Fig. 6.4 Relative increase of pressure corrected 2-min data of the neutron monitor stations Oulu, Apatity, Kiel and Rome during GLE\#70 (13 December 2006) plotted with NEST

In addition to the database there are different applications available from the NMDB webpage www.nmdb.eu (Mavromichalaki et al. 2011). NMDB provides e.g. the application NEST (http://www.nmdb.eu/nest/) to generate plots of the count rate of selected neutron monitor stations with different time resolutions. It is also possible to plot neutron monitor data together with the sunspot number (smoothed or monthly), geomagnetic $K p$-index (3-hourly), or GOES data (channels $>10 \mathrm{MeV}$, $>50 \mathrm{MeV},>100 \mathrm{MeV}$ ). The plots can be modified by different style adjustments. The generation of plots during GLEs and Forbush decreases from the past can be selected with one click. Figure 6.4 shows as an example the measured relative increase in the count rate of a selection of neutron monitors during GLE\#70 on 13 December 2006 as plotted with the NMDB NEST application. In addition to the graphic output it is also possible to extract ASCII data from NMDB with NEST. Other NMDB applications are e.g. GLE alarm systems or GLE characteristics determination.

In addition to NMDB the Cosmic Ray Station of the University of Oulu reactivated and operates the GLE database http://gle.oulu.fi/ where the neutron monitor data of the worldwide network during GLEs are stored and are made available for plotting and for downloading. 


\section{References}

Agostinelli, S., et al.: GEANT4a simulation toolkit. Nucl. Instrum. Methods Phys. Res. A 506, 250-303 (2003)

Belov, A.V., Struminsky, A.B.: Neutron monitor sensitivity to primary protons below $3 \mathrm{GeV}$ derived from data of ground level events. In: 25th International Cosmic Ray Conference, Durban, vol. 1, p. 201 (1987)

Bieber, J.W., Clem, J., Desilets, D., Evenson, P., Lal, D., Lopate, C., Pyle, R.: Long-term decline of South Pole neutron rates. J. Geophys. Res. (Space Phys.) 112, A12102 (2007)

Bütikofer, R., Flückiger, E.O.: Pressure Correction of GLE measurements in turbulent winds. Int. Cosmic Ray Conf. 6, 395 (1999)

Carmichael, H.: Cosmic Rays, IQSY Instruction Manual No. 7. IQSY Secretariat, London (1964)

Carmichael, H.: Annals of the IQSY. In: Minnis, C.M. (ed.) Geophysical Measurements: Techniques, Observational Schedules and Treatments of Data, vol. 1. MIT Press, Cambridge (1968)

Carmichael, H., Bercovitch, M., Shea, M.A., Magidin, M., Peterson, R.W.: Attenuation of neutron monitor radiation in the atmosphere. Can. J. Phys. Suppl. 46, 1006 (1968)

Clem, J.M., Dorman, L.I.: Neutron monitor response functions. Space Sci. Rev. 93, 335-359 (2000)

Debrunner, H., Lockwood, J.A., Flückiger, E.: Specific yield function $\mathrm{S}(\mathrm{P})$ for a neutron monitor at sea level, paper presented. In: 8th European Cosmic Ray Symposium, Rome (1982)

Desorgher, L., Flückiger, E.O., Gurtner, M.: The PLANETOCOSMICS Geant4 application. In: 36th COSPAR Scientific Assembly, vol. 36 of COSPAR Meeting, p. 2361 (2006)

Dorman, L.I., Yanke, V.G.: The coupling functions of NM-64 neutron supermonitor. Int. Cosmic Ray Conf. 4, 326 (1981)

Fassò, A., Ferrari, A., Ranft, J., Sala, P.R., Stevenson, G.R., Zazula, J.M.: A comparison of FLUKA simulations with measurements of fluence and dose in calorimeter structures. Nucl. Instrum. Methods Phys. Res. A 332, 459-468 (1993)

Flückiger, E.O., Moser, M.R., Pirard, B., Bütikofer, R., Desorgher, L.: A parameterized neutron monitor yield function for space weather applications. Int. Cosmic Ray Conf. 1, 289-292 (2008)

Hatton, C.J.: The Neutron Monitor. American Elsevier Publishing Company, New York (1971)

Hedin, A.E.: Extension of the MSIS thermosphere model into the middle and lower atmosphere. J. Geophys. Res. 96, 1159-1172 (1991)

Labitzke, K., Barnett, J.J., Edwards, B.: Handbook MAP, vol. 16 (1985)

Matthiä, D.: The radiation environment in the lower atmosphere: a numerical approach. Ph.D. thesis, Christian-Albrechts-Universität zu Kiel (2009)

Mavromichalaki, H., et al.: Applications and usage of the real-time neutron monitor database. Adv. Space Res. 47, 2210-2222 (2011)

McCracken, K.G.: The cosmic-ray flare effect, 1, some new methods of analysis. J. Geophys. Res. 67, 423-434 (1962)

Mishev, A.L., Usoskin, I.G., Kovaltsov, G.A.: Neutron monitor yield function: new improved computations. J. Geophys. Res. (Space Phys.) 118, 2783-2788 (2013)

Mishev, A.L., Kocharov, L.G., Usoskin, I.G.: Analysis of the ground level enhancement on 17 May 2012 using data from the global neutron monitor network. J. Geophys. Res. (Space Phys.) 119, 670-679 (2014)

Picone, J.M., Hedin, A.E., Drob, D.P., Aikin, A.C.: NRLMSISE-00 empirical model of the atmosphere: statistical comparisons and scientific issues. J. Geophys. Res. (Space Phys.) 107, 1468 (2002)

Pyle, R.: Public access to neutron monitor datasets. Space Sci. Rev. 93, 381-400 (2000)

Simpson, J.A.: The latitude dependence of neutron densities in the atmosphere as a function of altitude. Phys. Rev. 73, 1389-1391 (1948) 
Simpson, J.A.: Cosmic radiation neutron intensity monitor. Ann. Int. Geophys. Year 4, 351-373 (1957)

Shen, M.: Neutron production in lead and energy response of neutron monitor. Suppl. Nuovo Cimento 6, 1177 (1968)

Open Access This chapter is licensed under the terms of the Creative Commons Attribution 4.0 International License http://creativecommons.org/licenses/by/4.0/), which permits use, sharing, adaptation, distribution and reproduction in any medium or format, as long as you give appropriate credit to the original author(s) and the source, provide a link to the Creative Commons license and indicate if changes were made.

The images or other third party material in this chapter are included in the chapter's Creative Commons license, unless indicated otherwise in a credit line to the material. If material is not included in the chapter's Creative Commons license and your intended use is not permitted by statutory regulation or exceeds the permitted use, you will need to obtain permission directly from the copyright holder. 